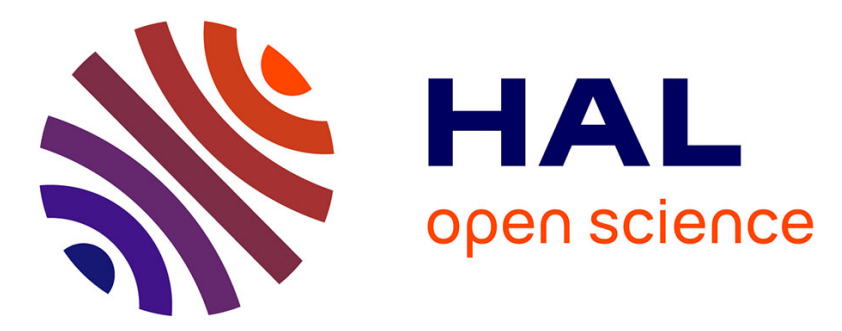

\title{
X-FEM, de nouvelles frontières pour les éléments finis
}

Nicolas Moës, Ted Belytschko

\section{To cite this version:}

Nicolas Moës, Ted Belytschko. X-FEM, de nouvelles frontières pour les éléments finis. 5e Colloque national en calcul des structures, CSMA, May 2001, Giens, France. pp.305 - 318, 10.3166/reef.11.305318. hal-01448481

\section{HAL Id: hal-01448481 \\ https://hal.science/hal-01448481}

Submitted on 28 Jan 2017

HAL is a multi-disciplinary open access archive for the deposit and dissemination of scientific research documents, whether they are published or not. The documents may come from teaching and research institutions in France or abroad, or from public or private research centers.
L'archive ouverte pluridisciplinaire HAL, est destinée au dépôt et à la diffusion de documents scientifiques de niveau recherche, publiés ou non, émanant des établissements d'enseignement et de recherche français ou étrangers, des laboratoires publics ou privés. 


\title{
$\mathrm{X}$-FEM, de nouvelles frontières pour les éléments finis
}

\author{
Nicolas Moës* - Ted Belytschko**
}

Laboratoire de Mécanique et Matériaux

École Centrale de Nantes

1 rue de la Noё, BP 92101

44321 Nantes Cedex 3

nicolas.moes@ec-nantes.fr

Northwestern University

Department of Mechanical Engineering

2145 Sheridan Rd, Evanston, IL 60208 USA

t-belytschko@nwu.edu

RÉSUMÉ. En 1999, une extension de la méthode des éléments finis a été proposée. Baptisée depuis $X$-FEM ("eXtended Finite Element Method"), cette extension permet de modéliser des surfaces de discontinuité (fissures, interfaces matériaux, bords libres, ...) sur un maillage sans que ce dernier doive s'y conformer. Ce papier dresse le bilan des avancées réalisées avec X-FEM; tout particulièrement le couplage de X-FEM avec la méthode des "level sets" qui permet une représention efficace des surfaces de discontinuité ainsi qu'un calcul robuste de leur évolution. ABSTRACT. In 1999, an extension of the finite element method was introduced. Later called $X$-FEM ("eXtended Finite Element Method"), this extension allows one to model surfaces of discontinuity (cracks, material interfaces, free surfaces, ...) on a mesh even if the mesh does not conform to these surfaces. This paper summarizes the achievement obtained so far with $X$ FEM, especially regarding the coupling of X-FEM with the "level sets" method which provides an efficient representation of the surfaces and a robust computation of their evolution. MOTS-CLÉS : éléments finis, $X$-FEM, discontinuités, partition de l'unité, méthode des "level sets", mécanique de la rupture, modélisation d'interfaces.

KEYWORDS: finite elements, $X$-FEM, discontinuities, partition of unity, level sets method, fracture mechanics, interface modeling. 


\section{Introduction}

La méthode des éléments finis est maintenant un outil standard pour la simulation numérique dans l'industrie. Avec la performance sans cesse croissante des ordinateurs, l'utilisation d'architectures parallèles et le développement d'algorithmes de résolution de plus en plus sophistiqués, le temps du calcul ne cesse de décroître et ce, à une vitesse vertigineuse. D'un autre côté, les mailleurs se sont améliorés. Les mailleurs 2D sont maintenant très robustes. Quant aux mailleurs 3D, des avancées ont été réalisées mais pas au point de ne plus nécessiter l'intervention fréquente de l'utilisateur. Ce décalage entre la difficulté de réaliser le maillage et l'aisance de plus en plus grande dans la résolution du modèle éléments finis conduit à des situations où le temps homme-machine requis pour la génération de maillage dépasse de loin le temps du calcul proprement dit.

La difficulté dans la génération du maillage est principalement due à la nécessité de ce dernier de respecter des surfaces : la surface extérieure bien sûr et des surfaces internes (trous, fissures, interfaces entre matériaux, ...). L'approximation éléments finis est en effet classiquement très régulière à l'intérieur des éléments et la modélisation de discontinuités dans la solution ne peut se faire qu'en bordure des éléments. En clair, les surfaces de discontinuité ne peuvent couper les éléments. Lorsque ces surfaces évoluent dans le temps (propagation de fissures, expansion de trous, propagation d'un front de solidification...), le remaillage est inévitable, de même que souvent, la projection de la solution entre l'ancien et le nouveau maillage. Le remaillage local est également nécessaire si une nouvelle surface est introduite dans un maillage existant. Par exemple, l'introduction d'une fissure dans un modèle éléments finis existant nécessite un remaillage local. En résumé, la simulation numérique d'un problème industriel implique en général non seulement la réalisation d'un maillage initial mais également bien souvent des remaillages. Outre les contraintes géométriques qui pèsent sur la génération du maillage, l'utilisateur souhaite aussi parfois imposer la taille des éléments dans les diverses zones du maillage ou remailler afin de réduire les erreurs d'approximation. En bref, le (re)maillage est bel et bien la pierre d'achoppement des calculs industriels.

Devant ce constat, une recherche soutenue a été effectuée depuis près de dix ans pour développer des méthodes dans lesquelles l'approximation ne repose pas sur un maillage mais sur un ensemble de points. En chaque point, des degrés de liberté sont définis. Ils agissent sur des fonctions de base à support compact autour du point. Différentes variantes existent à ce jour pour la construction des fonctions de base : les éléments diffus [NAY 92], la méthode "Element Free Galerkin" [BEL 94], la méthode "Reproducing Kernel Particle Method" [LIU 93] et la méthode " $h-p$ cloud" [DUA 96]. Ces approches dites "meshless" ou "meshfree" permettent en principe de se débarrasser de toutes les difficultés associées au maillage : la distribution des points ne doit pas respecter les surfaces intérieures ou extérieures de la structure, la présence de fissures ou interfaces entre matériaux est modélisée par l'introduction de fonctions discontinues [FLE 97] ou à dérivée discontinue [KRO 98], le remaillage n'est pas nécessaire lors de l'évolution des fissures [KRY 99]. Cette flexibilité s'ob- 
tient malheureusement à un prix élevé. Des opérations triviales dans le cadre éléments finis comme l'évaluation d'une fonction de forme en un point, l'intégration des formes bilinéaires et linéaires ou la prise en compte de conditions aux limites de type Dirichlet ne sont plus triviales et sont même coûteuses. De plus, les méthodes "meshfree" introduisent dans la construction de l'approximation des paramètres dont le choix approprié reste une question d'actualité.

Dans ce papier, nous décrivons une alternative pour se dégager des difficultés liées au maillage. Cette approche est baptisée X-FEM ("eXtended Finite Element Method"). Contrairement aux approches "meshfree" où le maillage est abandonné et avec lui malheureusement de nombreux ingrédients qui font la robustesse et la flexibilité de la méthode des éléments finis, nous optons pour la conservation du maillage mais nous le libérons des contraintes qui rendent sa génération si délicate : le respect scrupuleux de surface. La génération du maillage n'est alors plus contrainte que par la qualité de l'approximation numérique. Pour parvenir à ce but, nous utilisons une technique appelée partition de l'unité introduite par Melenk et Babuška (1996). Cette technique permet d'enrichir une approximation éléments finis à l'aide de fonctions quelconques tout en préservant le caractère creux de la matrice éléments finis globale. Dans le papier de Melenk Babuška (1996) et les travaux de Strouboulis et al. (2000a), (2000b), la partition de l'unité est utilisée essentiellement afin d'améliorer la qualité de l'approximation éléments finis par le rajout de solutions analytiques près de trous ou en pointe de fissure. La partition de l'unité a également été utilisée par Oden, Duarte et Zienkiewicz (1998) pour générer aisément une approximation $h-p$ non uniforme et robuste vis-à-vis de la distorsion des éléments. Ici, nous utilisons cette technique à une autre fin : libérer le maillage de sa servitude à des surfaces par le choix pertinent de l'enrichissement. Si la surface est celle d'un trou ou d'une frontière extérieure, la fonction pour enrichir est à valeur 1 dans la matière et 0 en dehors. Si la surface est une surface de discontinuité (ex. fissure), la fonction a pour valeur -1 d'un côté de la surface et 1 de l'autre. Enfin, si la surface est une surface de discontinuité dans la dérivée du champ (ex. interface entre matériau) la fonction est donnée par la distance à la surface. Suite à cet enrichissement, un élément peut contenir un vide en son sein ou un saut dans le champ ou dans sa dérivée. Il n'est plus nécessaire de faire coïncider le maillage avec les surfaces de discontinuité pour les modéliser. Le maillage est "libéré". L'extension X-FEM de la méthode des éléments finis a trois avantages importants. D'abord, elle est générale et non liée à un modèle de comportement particulier (élastique, plastique...). Elle se contente de représenter un saut dans le champ, sa dérivée ou la présence d'un vide dans l'élément. Ensuite, si le maillage respecte les surfaces de discontinuité, l'enrichissement n'a pas d'effet et le cadre classique éléments finis est retrouvé. Enfin, l'enrichissement est valable quelle que soit la dimension du problème : 1D, 2D ou 3D et ce quel que soit le type des éléments utilisés. L'utilisation de plusieurs types d'éléments sur un même maillage (triangles et quadrilatères ou tétraèdres, hexaèdres et prismes) ne pose aucune difficulté. En quelque sorte X-FEM étend les possibilités de la méthode des éléments finis sans en perdre les avantages. Bien sûr, des informations associées à une situation particulière peuvent être incorporées pour améliorer la précision du calcul. Par exemple, pour des simulations élastiques, 
nous utilisons les caractéristiques asymptotiques du champ de déplacement en pointe de fissure dans l'enrichissement tout comme dans les travaux de Strouboulis, Babuška et Copps (2000) .

Notons que l'introduction de fonctions discontinues à l'intérieur même des éléments finis n'est pas nouvelle. Déjà dans les travaux de Belytschko et al (1988) et Oliver (1995), cette idée apparait. Cependant, l'introduction se fait malheureusement par le biais d'une formulation mixte nécessitant la vérification de conditions de stabilité (conditions de type LBB) et conduit à une matrice globale non définie positive [JIR 00]. Une autre méthode, dite Arlequin [BEN 98], permet également d'introduire la présence d'une fissure sur un maillage grossier par le biais d'un maillage fin superposé au maillage grossier.

Avec la méthode X-FEM, le maillage ne doit plus se conformer aux surfaces extérieures et intérieures de la structure. Une conséquence importante est le fait que dans le calcul éléments finis la représentation de ces surfaces n'est plus donnée par un ensemble de faces d'éléments. Comment alors les représenter? Nous avons introduit deux approches. La première, que nous qualifions de lagrangienne, consiste à représenter les surfaces par en ensemble d'entités de base telles que celles que fournirait un modeleur géométrique. La seconde que nous qualifions d'eulérienne, utilise la méthode des "level sets" [SET 99]. Dans cette méthode, la surface est remplacée par une fonction de niveau ("level set") qui donne en tout point la distance à la surface et utilise le signe placé devant cette distance pour indiquer si le point se trouve d'un côté ou de l'autre de la surface. En pratique, la fonction de niveau est calculée aux nœuds du maillage et les fonctions de base éléments finis interpolent ces valeurs. Cette représentation par fonctions de niveau des surfaces facilite la mise en œuvre de X-FEM qui necessite des opérations de type géométrique pour l'évaluation des fonctions d'enrichissement : distance à une surface, normale à une surface, savoir si l'on se trouve d'un côté ou de l'autre d'une surface... Ces opérations géométriques reviennent respectivement à évaluer la fonction de niveau, sa dérivée et son signe. Par contre, la représentation lagrangienne conduit à une implémentation plus coûteuse et laborieuse de ces opérations géométriques. Un autre avantage de la méthode des "level sets" (avantage qui fut à la base de son succès) est de permettre le calcul robuste de l'évolution d'une surface soumise à un champ de vitesse normale [SET 99], [OSH 88] .

La mécanique de la rupture est un domaine intéressant pour appliquer X-FEM. La fissure est une surface à travers laquelle le champ de déplacement est discontinu. Sa modélisation classique par éléments finis impose au maillage de se conformer à la géométrie des lèvres de la fissure. En cas de propagation, le remaillage est nécessaire. Les travaux de Rashid (1998) et de Ingraffea et al. [GER 87], [MAR 93] ont contribué à la simplification de ces remaillages, mais ils restent laborieux spécialement en 3D. La modélisation complète de fissures avec X-FEM a été présentée dans [MOË 99] et étendue dans [SUK 00] au cas tridimensionnel. Sukumar et al. [SUK 01b], ont ensuite couplé X-FEM à la "fast marching method" [SET 99] pour résoudre des problèmes de fissuration plane dans des milieux 3D. Enfin, la propagation non plane de fissures dans 
des milieux 3D a été réalisée dans [GRA 02]. La méthode X-FEM a également été appliquée à d'autres domaines de la mécanique décrits dans les sections qui suivent.

Le reste de ce papier est organisé comme suit. La section 2 résume la technique de la partition de l'unité, technique vitale pour X-FEM. La section 3 décrit la modélisation de fissures par X-FEM et leur représentation à l'aide de la méthode des "level sets". La section suivante concerne la modélisation de trous, bords libres et la dernière section, la modélisation d'interfaces matériaux.

\section{La partition de l'unité}

Dans la méthode X-FEM, les surfaces de discontinuité sont modélisées par un enrichissement de la base éléments finis à l'aide la technique dite de la partition de l'unité introduite par Melenk et Babuška (1996). Cette technique est résumée ci-dessous. L'approximation éléments finis (d'un champ de déplacement pour fixer les idées) sur un élément, $\Omega_{e}$, s'écrit :

$$
\left.\boldsymbol{u}(\boldsymbol{x})\right|_{\Omega_{e}}=\sum_{i \in N_{n}(\boldsymbol{x})} \sum_{\alpha} a_{i}^{\alpha} \phi_{i}^{\alpha}(\boldsymbol{x})
$$

où $N_{n}(\boldsymbol{x})$ est l'ensemble des nœuds de l'élément contenant le point $\boldsymbol{x} ; u_{i}^{\alpha}$ est le déplacement au nœud $i$ dans la direction $\alpha$ et $\phi_{i}^{\alpha}$ est la fonction de forme correspondante. Comme les degrés de liberté définis en un nœud ont la même valeur pour tous les éléments connectés à ce nœud, les approximations sur chaque élément peuvent être "assemblées" pour donner une approximation valable en tout point $\boldsymbol{x}$ du domaine $\Omega$ :

$$
\boldsymbol{u}(\boldsymbol{x})=\sum_{i \in N_{n}(\boldsymbol{x})} \sum_{\alpha} a_{i}^{\alpha} \phi_{i}^{\alpha}(\boldsymbol{x})
$$

Le domaine d'influence (support) de la fonction d'interpolation $\phi_{i}^{\alpha}$ est l'ensemble des éléments connectés au nœud $i$. L'ensemble $N_{n}(\boldsymbol{x})$ est donc également l'ensemble des nœuds dont le support couvre le point $\boldsymbol{x}$. L'approximation éléments finis enrichie qui permet de représenter un déplacement d'amplitude $F(\boldsymbol{x})$ selon n'importe quelle direction sur le domaine $\Omega_{F} \subset \Omega$ s'écrit :

$$
\boldsymbol{u}(\boldsymbol{x})=\sum_{i \in N_{n}(\boldsymbol{x})} \sum_{\alpha} \boldsymbol{\phi}_{i}^{\alpha} a_{i}^{\alpha}+\sum_{i \in N_{n}(\boldsymbol{x}) \cap N_{F}} \sum_{\alpha} b_{i}^{\alpha} \boldsymbol{\phi}_{i}^{\alpha}(\boldsymbol{x}) F(\boldsymbol{x})
$$

où $N_{F}$ est l'ensemble des nœuds dont le support a une intersection avec le domaine $\Omega_{F}$. La preuve est obtenue en fixant à zéro les coefficients $a_{i}^{\alpha}$ et en prenant en compte le fait que les fonctions de forme éléments finis sont capables de représenter tous les modes rigides.

\section{La modélisation de fissures avec X-FEM}

La modélisation d'une fissure par X-FEM comprend donc deux types d'enrichissement : un enrichissement pour le front de la fissure à l'aide de fonctions caractéri- 
sant le comportement asymptotique du champ de déplacement près du front de fissure [BEL 99] et un enrichissement pour l'intérieur de la fissure à l'aide d'une fonction à valeur 1.0 au-dessus de la fissure et -1.0 en dessous [MOË 99]. Le fait qu'un nœud soit enrichi ou non et le type d'enrichissement dépend de la position relative de son support par rapport à la fissure.
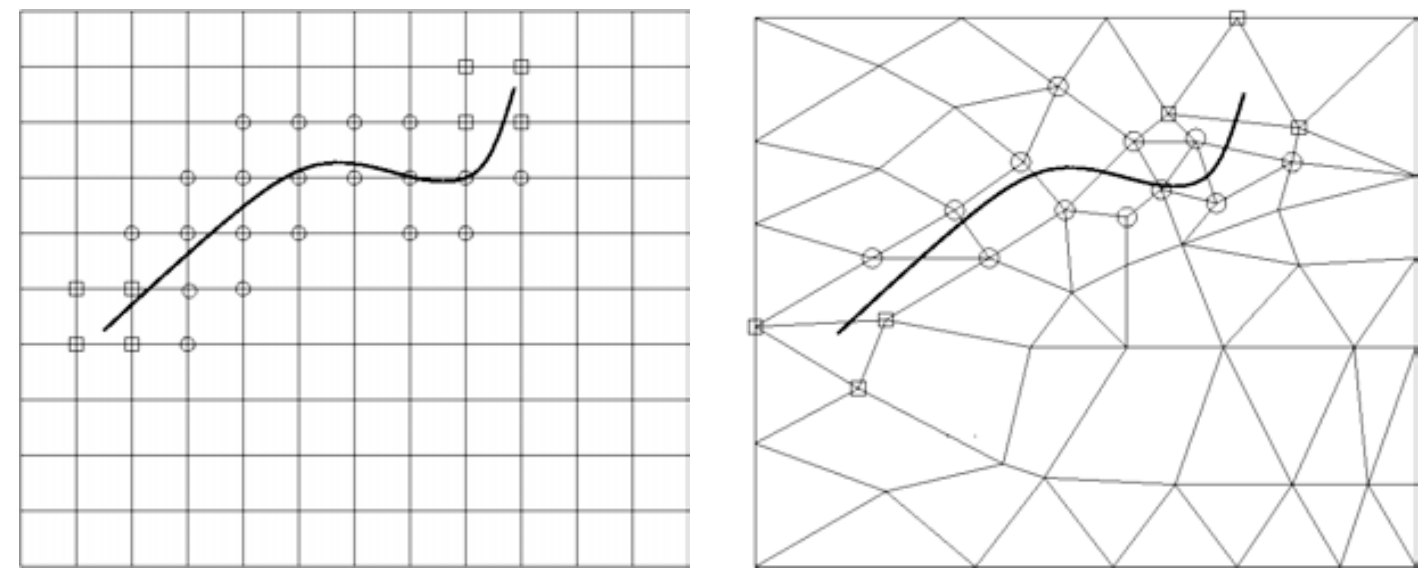

Figure 1. Fissure placée sur un maillage uniforme (gauche) ou non uniforme (droite). Les nœuds encerclés sont enrichis par la discontinuité et les nouds entourés d'un carré sont enrichis par les modes asymptotiques en fond de fissure

L'approximation éléments finis enrichie pour modéliser la présence d'une fissure s'écrit :

$$
\begin{aligned}
\boldsymbol{u}^{h}(\boldsymbol{x}) & =\sum_{i \in I} \boldsymbol{u}_{i} \phi_{i}(\boldsymbol{x})+\sum_{i \in L} \boldsymbol{a}_{i} \phi_{i}(\boldsymbol{x}) H(\boldsymbol{x}) \\
& +\sum_{i \in K_{1}} \phi_{i}(\boldsymbol{x})\left(\sum_{l=1}^{4} \boldsymbol{b}_{i, 1}^{l} F_{1}^{l}(\boldsymbol{x})\right)+\sum_{i \in K_{2}} \phi_{i}(\boldsymbol{x})\left(\sum_{l=1}^{4} \boldsymbol{b}_{i, 2}^{l} F_{2}^{l}(\boldsymbol{x})\right)
\end{aligned}
$$

où :

- $I$ est l'ensemble des nœuds du maillage;

- $\boldsymbol{u}_{i}$ est le degré de liberté (vectoriel) classique au nœud $i$;

$-\phi_{i}$ est la fonction de forme (scalaire) associée au nœud $i$;

- $L \subset I$ est l'ensemble des nœuds enrichis par la discontinuité et les coefficients $\boldsymbol{a}_{i}$ sont les degrés de liberté (vectoriels) correspondants. Un nœud appartient à $L$ si son support est coupé par la fissure mais ne contient aucune de ses pointes (ces nœuds sont encerclés sur la figure 3 dans le cas d'un maillage uniforme et non uniforme);

$-K_{1} \subset I$ et $K_{2} \subset I$ sont les ensembles des nœuds à enrichir pour modéliser les fonds de fissure 1 et 2, respectivement. Les degrés de liberté correspondants sont $\boldsymbol{b}_{i, 1}^{l}$ et $\boldsymbol{b}_{i, 2}^{l}, l=1, \ldots, 4$. Un nœud appartient à $K_{1}\left(K_{2}\right)$ si son support contient la première (seconde) pointe de fissure. Ces nœuds sont entourés d'un carré figure 3. 
Les fonctions $F_{1}^{l}(\boldsymbol{x}), l=1, \ldots, 4$ modélisant le fond de fissure sont données en élasticité par :

$$
\left\{F_{1}^{l}(\boldsymbol{x})\right\} \equiv\left\{\sqrt{r} \sin \left(\frac{\theta}{2}\right), \sqrt{r} \cos \left(\frac{\theta}{2}\right), \sqrt{r} \sin \left(\frac{\theta}{2}\right) \sin (\theta), \sqrt{r} \cos \left(\frac{\theta}{2}\right) \sin (\theta)\right\}
$$

où $(r, \theta)$ sont les coordonnées polaires dans des axes locaux en fond de fissure.

L'extension au cas tridimensionnel a été réalisée dans [SUK 00]. L'extension aux plaques fissurées pour le modèle de Reissner-Mindlin a été réalisée dans [DOL 00a], [DOL 00b]. Lorsque plus de deux branches aboutissent en un point de la fissure, (fissures en $\mathrm{Y}$ ou en croix), un soin particulier est à apporter à l'enrichissement. Cette extension a été réalisée dans [DAU 00]. En un point où plus de deux branches aboutissent, l'enrichissement fait intervenir une fonction dite de jonction.

On constate que la définition de l'approximation éléments finis enrichie nécessite un certains nombre d'opérations géométriques :

- un nœud est enrichi ou non selon la position respective de son support par rapport à la fissure.

- pour évaluer les fonctions $H(\boldsymbol{x})$ et $F(\boldsymbol{x})$, il faut savoir si le point $\boldsymbol{x}$ se trouve au dessus ou en dessous de la fissure ainsi que sa position $(r, \theta)$ par rapport au front de la fissure. Soit $\boldsymbol{x}^{*}$ Le point de la fissure le plus proche du point $\boldsymbol{x}$, on dit que $\boldsymbol{x}$ est situé au dessus (en dessous) de la fissure si le vecteur $\boldsymbol{x}-\boldsymbol{x}^{*}$ se projete positivement (négativement) sur la normale à la fissure au point $\boldsymbol{x}^{*}$.

- lors de l'intégration de la matrice de raideur sur les éléments coupés par la fissure, il faudra intégrer de part et d'autre de cette fissure.

Ces opérations sont réalisées facilement si la fissure est représentée à l'aide de deux fonctions de niveau ("level sets"). À chaque nœud du maillage, on associe deux nombres, le premier indique la distance à la fissure (plus précisement une extension tangente de celle-ci) compté positivement ou négativement selon que le nœud considéré se trouve d'un côté ou de l'autre de la fissure. Le second nombre indique la position du nœud par raport au front de la fissure. Les deux fonctions de niveau interpolent ces valeurs nodales à l'aide des fonctions de forme éléments finis classiques. Cette représentation des fissures à l'aide de fonctions de niveau a été introduite dans [STO 01] pour le cas $2 \mathrm{D}$ et dans [MOË 02] pour le cas 3D. La figure 3 illustre la représentation d'une fissure à l'aide de deux (en fait trois car il y a ici deux fronts de fissure) fonctions de niveau.

Dans le cas d'une fissure circulaire placée dans un cube, figure 3. La première fonction de niveau représente la distance au plan de la fissure (comptée positivement au dessus et négativement en dessous de la fissure) et la seconde fonction de niveau est la distance au cylindre passant par le front de la fissure (comptée positivement à l'extérieur et négativement à l'intérieur du cylindre).

Non seulement, la représentation d'une fissure par fonctions de niveau permet d'effectuer de manière rapide et robuste les opérations géométriques nécessaires à l'éva- 


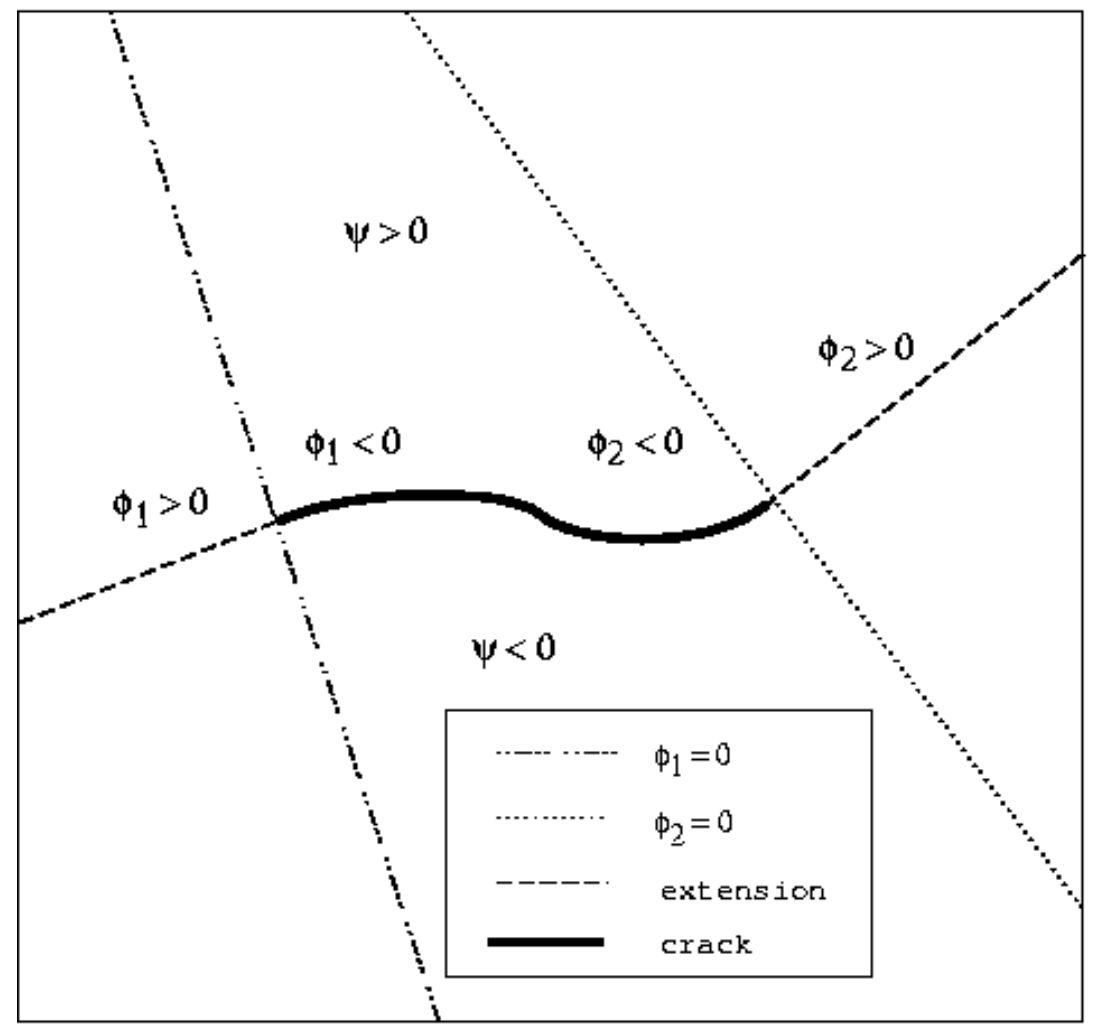

Figure 2. Les fonctions de niveau permettant de situer une fissure à deux fronts sur un maillage

luation des fonctions d'enrichissement mais elle permet également de gérer la propagation de la fissure [GRA 02]. La figure 4 donne la dernière étape de propagation de la fissure circulaire par fatigue.

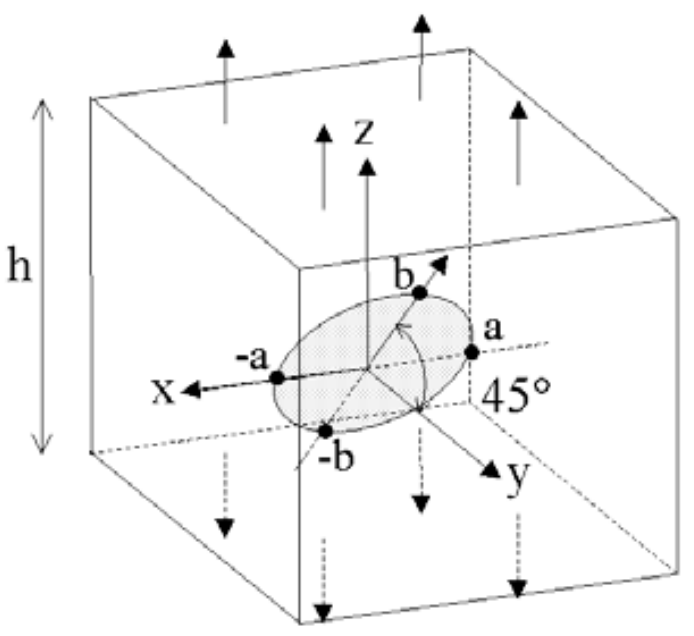

Figure 3. Une fissure circulaire inclinée dans un cube sous tension

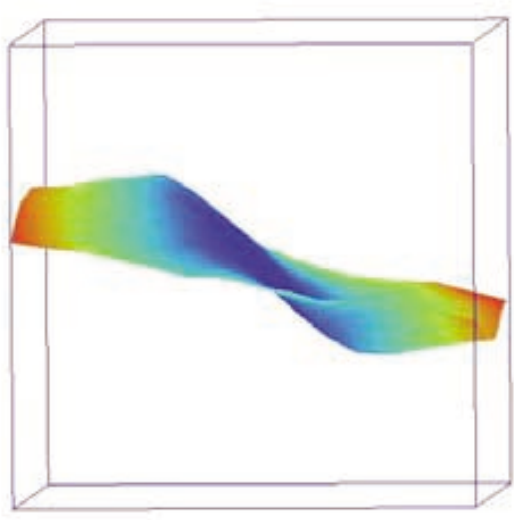

Figure 4. Position de la fissure à la dernière étape de sa propagation 
Comme autre exemple, considérons le problème d'un tunnel creusé dans une roche avec joints, figure 5. Ce problème met en jeu à la fois des surfaces de discontinuité et des bords libres et a été traité dans [BEL 01]. Le maillage utilisé, très simple, est montré figure 6. Il ne respecte ni la position du tunnel, ni la position des joints. L'enrichissement au niveau des joints ne fait intervenir qu'une discontinuité tangentielle. La figure 7 montre le déplacement horizontal obtenu.

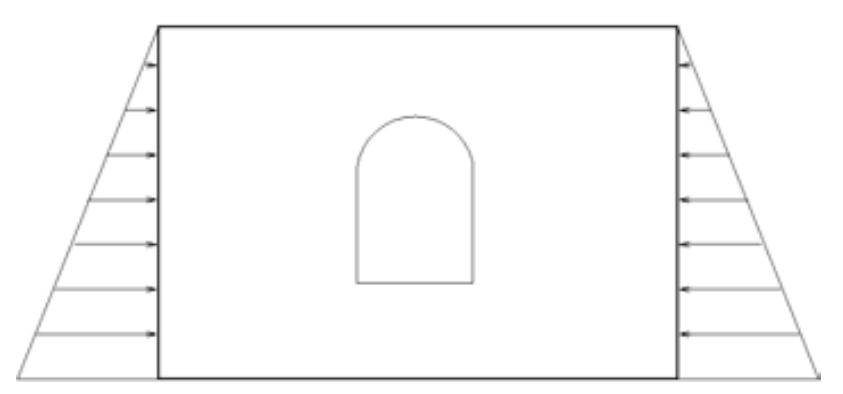

Figure 5. Représentation schématique du "jointed rocks problem"

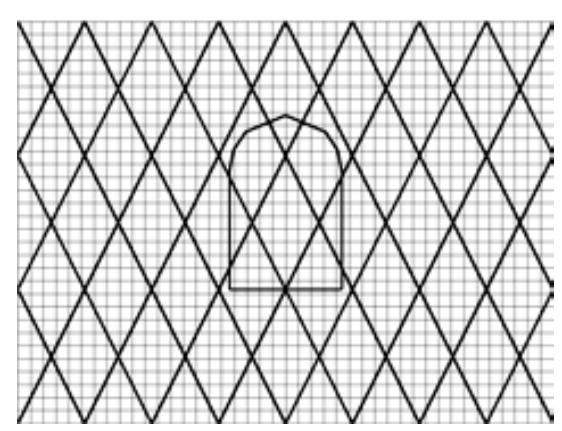

Figure 6. Le maillage utilisé avec la position des joints et du tunnel
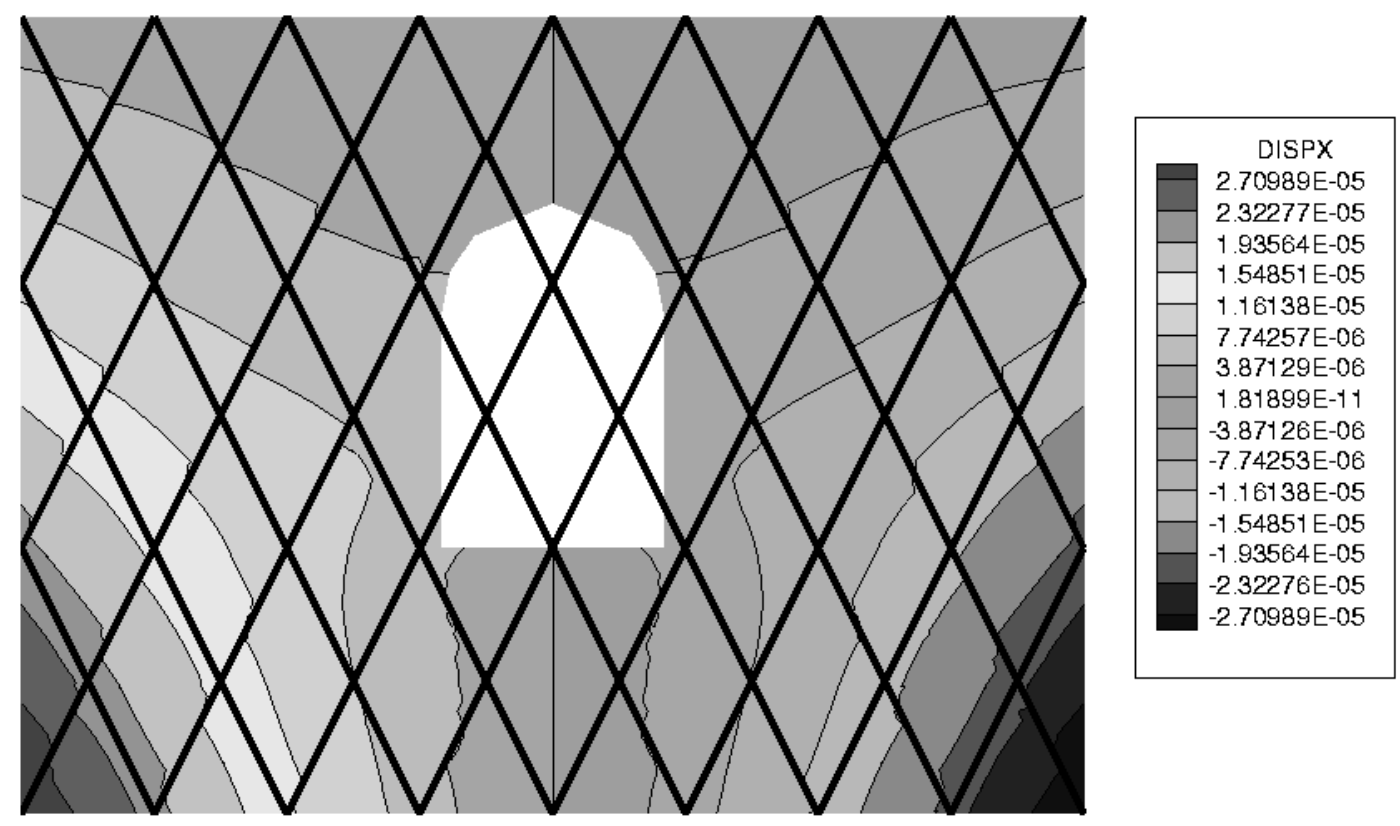

Figure 7. Déplacement horizontal discontinu au niveau des joints

Lorsque la fissure est en compression, la prise en compte du contact et de la friction devient nécessaire. Un couplage entre X-FEM et la méthode LATIN [LAD 96] permet 
de modéliser le contact et la friction sur les lèvres de la fissure [DOL 01]. Il faut noter que contrairement au traitement classique du contact où il faut distinguer le maillage d'un côté et de l'autre de la fissure, un seul et unique maillage sur lequel la fissure est "posée" est utilisé dans X-FEM. Les difficultés associées à des maillages non compatibles de part et d'autre de la zone de contact disparaissent. Finalement, il faut aussi noter la possibilité de la prise en compte d'une zone cohésion en pointe de fissure pour examiner les effets d'échelle dans les bétons [WEL 01], [MOË ] (à paraître).

\section{La modélisation de trous et bords libres}

La modélisation classique de trous (ou surfaces extérieures) par la méthode des éléments finis impose au maillage de se conformer à la frontière de ces trous. La méthode X-FEM permet de se débarrasser de cette contrainte. Pour un nœud dont le support coupe la frontière du trou, la fonction de forme classique est multipliée par une fonction valant 1.0 dans la matière et 0.0 dans les trous. Un nœud dont le support est complètement à l'intérieur du trou ne donne pas lieu à la création de degrés de liberté. Il est à noter qu'un nœud peut être actif même si il est situé dans un trou. Ce qui importe est le fait que son support couvre de la matière ou non. La figure 8 illustre la sélection des nœuds à éliminer et les nœuds pour lesquels la fonction de forme doit être modifiée [DAU 00].

C'est cette stratégie qui a permis de modéliser le tunnel dans l'exemple de la section précédente. On peut montrer que bien que le maillage ne se conforme pas au bord du trou, la convergence reste en $(O(h))$ pour des éléments du premier degré moyennant une intégration numérique ad hoc. Autrement dit la convergence éléments finis classique n'est pas affectée [SUK 01a].

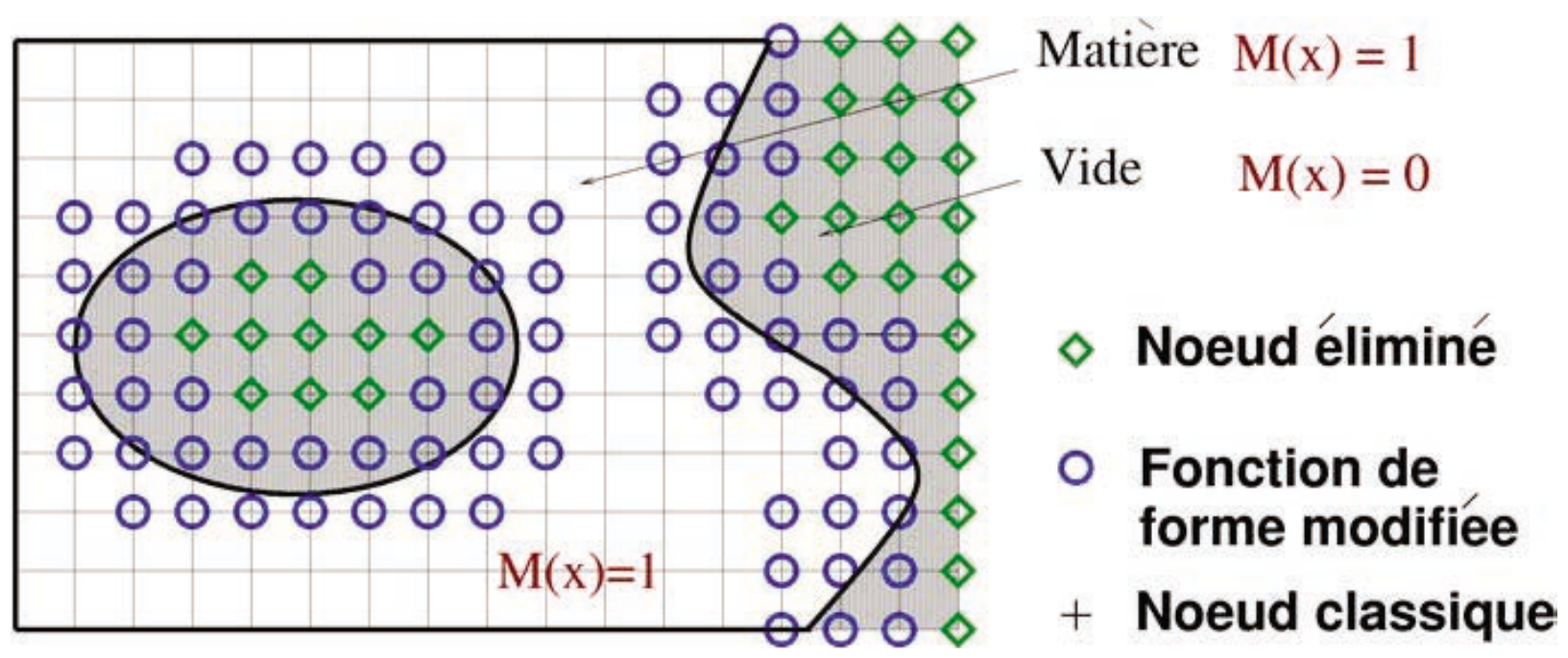

Figure 8. Sélection des nœuds pour le traitement des trous et surfaces libres dans $X$-FEM 


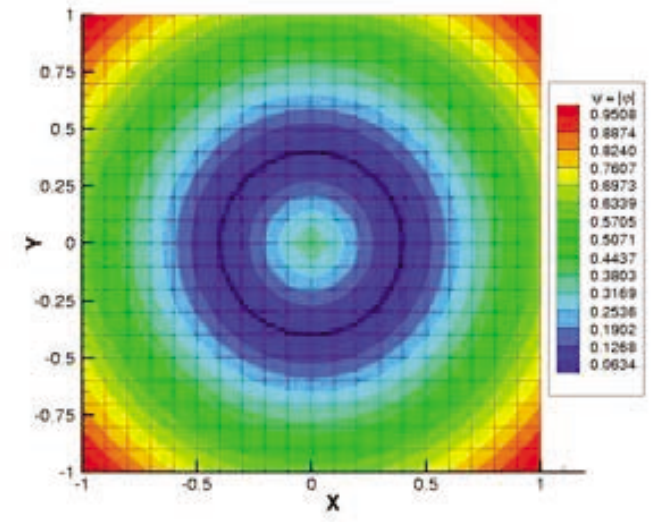

Figure 9. Valeur absolue de la fonction de niveau associée à une interface entre matériaux circulaire. Le niveau 0 correspond à la position de l'interface (cercle noir)

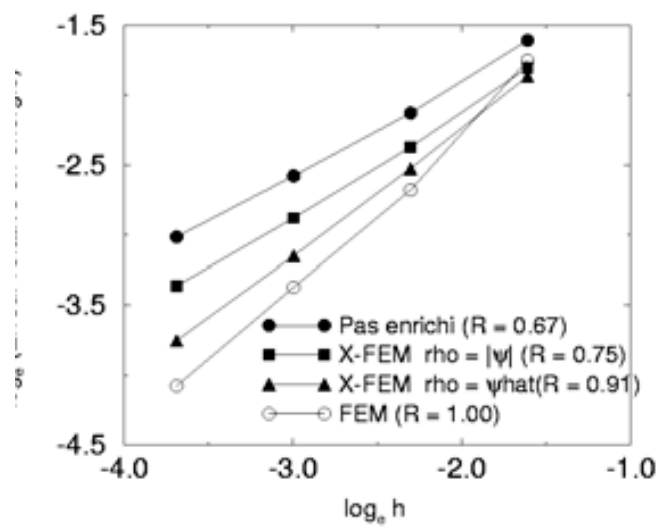

Figure 10. Convergence de l'erreur en énergie pour le problème de l'inclusion circulaire

\section{La modélisation d'interfaces entre matériaux}

Une interface entre matériaux conduit en mécanique à un champ de déplacement à dérivée discontinue au travers de l'interface. Lorsque le maillage respecte l'interface, la déformation est discontinue au travers de l'interface par le fait de la faible régularité $\left(C^{0}\right)$ de l'approximation éléments finis. Lorsque le maillage ne respecte pas l'interface, la base éléments finis doit être enrichie à l'aide d'une fonction à dérivée discontinue sur l'interface.

La valeur absolue de la fonction de niveau associée à l'interface (c'est à dire la distance à l'interface) joue parfaitement ce rôle. car elle est à dérivée discontinue sur l'interface. La figure 9 montre la valeur absolue de la fonction de niveau associée à une inclusion circulaire dans une plaque carrée en tension.

L'approximation éléments finis enrichie s'écrit :

$$
\boldsymbol{u}^{h}(\boldsymbol{x})=\sum_{i \in I} \boldsymbol{u}_{i} \phi_{i}(\boldsymbol{x})+\sum_{i \in D} \boldsymbol{a}_{i} \phi_{i}(\boldsymbol{x}) \rho(\boldsymbol{x})
$$

où $D$ est l'ensemble des nœuds dont le support est coupé par l'interface entre matériaux et $\rho(\boldsymbol{x})$ est une fonction à dérivée discontinue sur l'interface (la distance à l'interface).

Des maillages emboités ont été utilisés pour étudier la convergence de X-FEM. Les résultats sont donnés figure 10 . On constate que la convergence de la méthode des éléments finis classique (notée "Pas enrichi" sur la figure) est faible lorsque le maillage ne respecte pas la géométrie de l'inclusion : le taux de convergence est $R=0.67$. Lorsque le maillage se conforme à l'inclusion, la convergence est de 1.00 , comme 
annoncée par la théorie (noté FEM sur la figure). La convergence de X-FEM avec un enrichissement correspondant à la distance à l'interface est de $R=075$ et avec une fonction d'enrichisement lissée de $=091$ c'est-à-dire proche de la convergence optimale de 1.00 [SUK 01a]. Dans les problèmes de solidification, la conductivité thermique est discontinue sur le front et conduit à un champ de température à dérivée discontinue. Les travaux [JI ] et [CHE 02] utilisent X-FEM pour modéliser cette discontinuité et la méthode des "level sets" pour propager le front. Finalement, notons l'utilisation de X-FEM pour le calcul de l'écoulement de Stokes autour de particules rigides en mouvement [WAG 01].

\section{Conclusions}

L'extension de la méthode des éléments finis, baptisée X-FEM, permet de modéliser une discontinuité placée de manière quelconque sur un maillage. Cette discontinuité peut intervenir dans le champ, dans sa dérivée ou dans la matière (présence de trous ou de bords libres). La localisation de la surface de discontinuité se fait par le biais d'une ou plusieurs fonctions de niveau définies sur le maillage. La méthode des "level sets" permet de propager cette surface sous un champ de vitesse connu. Les applications traitées par X-FEM jusqu'ici concernent principalement la mécanique de la rupture et la modélisation d'interfaces entre matériaux différents. On peut espérer que son champ d'application s'étendra dans le futur.

\section{Bibliographie}

[Bel 88] Belytschko T., Fish J., Englemann B., « A finite element method with embedded localization zones ", Comp. Meth. in Applied Mech. and Engrg., vol. 70, 1988, p. 59-89.

[BEL 94] BelytschKo T., Lu Y., Gu L., « Element-free Galerkin methods », International Journal for Numerical Methods in Engineering, vol. 37, 1994, p. 229-256.

[BEL 99] Belytschko T., Black T., « Elastic Crack Growth in Finite Elements with Minimal Remeshing », International Journal for Numerical Methods in Engineering, vol. 45, $\mathrm{n}^{\mathrm{o}} 5,1999$, p. 601-620.

[Bel 01] Belytschko T., Mö̈s N., Usui S., Parimi C., « Arbitrary discontinuities in finite elements ", International Journal for Numerical Methods in Engineering, vol. 50, 2001, p. 993-1013.

[BEN 98] Ben DHIA H., «Problèmes mécaniques multi-échelles : la méthode Arlequin. », Compte-Rendus Acad. Sci. Paris, Série II, vol. 326, 1998, p. 899-904.

[CHE 02] Chessa J., S molinski P., Belytschko T., « The extended finite element method (XFEM) for solidification problems », International Journal for Numerical Methods in Engineering, vol. 53, 2002, p. 1959-1977.

[DAU 00] Daux C., Moës N., Dolbow J., Sukumar N., Belytschko T., « Arbitrary branched and intersecting cracks with the eXtended Finite Element Method», International Journal for Numerical Methods in Engineering, vol. 48, 2000, p. 1741-1760. 
[DOL 00a] Dolbow J., Mö̈s N., Belytschko T., « Discontinuous Enrichment in Finite Elements with a Partition of Unity Method », Finite elements in analysis and design, vol. 36, 2000, p. 235-260.

[DOL 00b] Dolbow J., Mö̈s N., Belytschko T., « Modeling fracture in MindlinReissner plates with the eXtended finite element method », Int. J. Solids Structures, vol. 37, 2000, p. 7161-7183.

[DOL 01] Dolbow J., MoËs N., Belytschko T., «An extended finite element method for modeling crack growth with frictional contact », Comp. Meth. in Applied Mech. and Engrg., vol. 190, 2001, p. 6825-6846.

[DUA 96] DUARTE C., OdEN J., « An hp meshless method », Numerical methods for partial differential equations, vol. 12, 1996, p. 673-705.

[Fle 97] Fleming M., Chu Y. A., Moran B., Belytschko T., « Enriched element-free Galerkin methods for crack tip fields », International Journal for Numerical Methods in Engineering, vol. 40, $\mathrm{n}^{\circ}$ 8, 1997, p. 1483-1504.

[GER 87] Gerstle W. H., Martha L., IngraffeA A. R., « Finite and Boundary Element Modeling of Crack Propagation in Two- and Three-Dimensions », Engineering with Computers, vol. 2, 1987, p. 167-183.

[GRA 02] Gravouil A., Mö̈s N., Belytschko T., " Non-planar 3D crack growth by the extended finite element and level sets. Part II : level set update », International Journal for Numerical Methods in Engineering, vol. 53, 2002, p. 2569-2586.

[JI ] Ji H., Chopp D., Dolbow J., « A hybrid extended finite element / level set method for modeling phase transformation ", International Journal for Numerical Methods in Engineering, to appear.

[JIR 00] JIRÁSEK M., " Comparative study on finite elements with embedded discontinuities », Comp. Meth. in Applied Mech. and Engrg., vol. 188, $\mathrm{n}^{\circ}$ 1, 2000, p. 307-330.

[KRO 98] KRONGAUZ Y., BELYTSCHKO T., «EFG approximation with discontinuous derivatives », International Journal for Numerical Methods in Engineering, vol. 41, $\mathrm{n}^{\circ}$ 7, 1998, p. $1215-1233$.

[KRY 99] KRYSL P., BELYTSCHKO T., «Element free Galerkin method for dynamic propagation of arbitrary 3-D cracks », International Journal for Numerical Methods in Engineering, vol. 44, n 6,1999 , p. 767-800.

[LAD 96] LADEVÈzE P., Mécanique non linéaire des structures. Nouvelle approche et méthodes de calcul non incrémentales, Hermes, 1996, (English version, Springer 1998).

[LIU 93] LiU W., Adee J., Jun S., Belytschko T., « Reproducing Kernel Particle Methods for elastic and plastic problems », Am Soc Mech Eng Appl Mech Div AMD, vol. 180, 1993, p. $175-189$.

[MAR 93] Martha L. F., WAWrzyneK P. A., IngraffeA A. R., « Arbitrary crack representation using solid modeling », Engineering with Computers, vol. 9, 1993, p. 63-82.

[MEL 96] Melenk J., BABUŠKa I., « The Partition of Unity Finite Element Method : Basic theory and applications », Comp. Meth. in Applied Mech. and Engrg., vol. 39, 1996, p. 289-314.

[MOË ] MoËs N., Belytschko T., « Extended Finite Element Method for Cohesive Crack Growth », Engineering Fracture Mechanics, To appear. 
[MOË 99] Mö̈s N., Dolbow J., Belytschko T., « A finite element method for crack growth without remeshing », International Journal for Numerical Methods in Engineering, vol. 46, 1999, p. 131-150.

[MOË 02] Mö̈s N., GRAVOuIl A., BelytschKo T., « Non-planar 3D crack growth by the extended finite element and level sets. Part I : Mechanical model », International Journal for Numerical Methods in Engineering, vol. 53, 2002, p. 2549-2568.

[NAY 92] Nayroles B., Touzot G., Villon P., « Generalizing the finite element method: Diffuse approximation and diffuse elements », Computers and Structures, vol. 10, $\mathrm{n}^{\mathrm{O}} 5$, 1992, p. 307-318.

[ODE 98] Oden J., Duarte C., Zienkiewicz O., « New cloud-based hp finite element method », Comp. Meth. in Applied Mech. and Engrg., vol. 153, n 1, 1998, p. 117-126.

[OLI 95] OLIVER J., « Continuum modeling of strong discontinuities in solid mechanics using damage models », Computational Mechanics, vol. 17, 1995, p. 49-61.

[OSH 88] OSher S., Sethian J. A., « Fronts Propagating with Curvature-Dependent Speed : Algorithms Based on Hamilton-Jacobi Formulations », Journal of Computational Physics, vol. $79, \mathrm{n}^{\circ} 1,1988$, p. 12-49.

[RAS 98] RASHID M. M., « The arbitrary local mesh refinement method : An alternative to remeshing for crack propagation analysis », Comp. Meth. in Applied Mech. and Engrg., vol. 154,1998, p. 133-150.

[SET 99] Sethian J. A., Level Set Methods \& Fast Marching Methods : Evolving Interfaces in Computational Geometry, Fluid Mechanics, Computer Vision, and Materials Science, Cambridge University Press, Cambridge, UK, 1999.

[STO 01] Stolarska M., Chopp D. L., Moës N., BelytschKo T., « Modelling Crack Growth by Level Sets and the Extended Finite Element Method », International Journal for Numerical Methods in Engineering, vol. 51, $\mathrm{n}^{\circ}$ 8, 2001, p. 943-960.

[STR 00] Strouboulis T., BABUŠKA I., COPPS K., " The design and analysis of the generalized finite element method », Comp. Meth. in Applied Mech. and Engrg., vol. 181, 2000, p. $43-71$.

[SUK 00] Sukumar N., Mö̈s N., Belytschko T., Moran B., « Extended Finite Element Method for three-dimensional crack modelling », International Journal for Numerical Methods in Engineering, vol. 48, $\mathrm{n}^{\mathrm{O}}$ 11, 2000, p. 1549-1570.

[SUK 01a] Sukumar N., Chopp D. L., MoËs N., Belytschko T., « Modeling Holes and Inclusions by Level Sets in the Extended Finite Element Method », Comp. Meth. in Applied Mech. and Engrg., vol. 190, 2001, p. 6183-6200.

[SUK 01b] Sukumar N., Chopp D. L., Moran B., « Extended Finite Element Method and Fast Marching Method for Three-Dimensional Fatigue Crack Propagation », Engineering Fracture Mechanics, , 2001, submitted.

[WAG 01] Wagner G., Mö̈s N., LiU W., Belytschko T., « The Extended Finite Element Method for Stokes Flow Past Rigid Cylinders », International Journal for Numerical Methods in Engineering, vol. 51, 2001, p. 393-413.

[WEL 01] Wells G., SluYs L., «A new method for modelling cohesive cracks using finite elements », International Journal for Numerical Methods in Engineering, vol. 50, 2001, p. 2667-2682. 